\title{
Hypertrophic obstructive cardiomyopathy
}

\section{Background}

Hypertrophic obstructive cardiomyopathy (HOCM), also known as hypertrophic cardiomyopathy (HCM), is an inherited condition that affects the muscle of the heart. It is the most common cause of sudden cardiac arrest and death in young athletes. It is caused by mutations in different genes that affect the structure, function and organization of heart muscle cells. This causes the heart to become abnormally thick, particularly at the septum between the left and right sides of the heart, which may cause obstruction of blood flow from the heart during each contraction. Thickening of the heart muscle may also be seen in other cardiovascular conditions such as high blood pressure and narrowing of the aortic valve, known as aortic stenosis. Early diagnosis is often difficult, as some individuals with HOCM don't experience symptoms. Once diagnosed, appropriate treatment is key to relieving symptoms and preventing serious complications, which include abnormal heart rhythms, heart failure and sudden death.

\section{Signs and symptoms}

While some individuals don't experience symptoms, others may experience chest pain, shortness of breath or fainting, especially during periods of exertion. Fainting suggests that the outflow of blood from the heart may be obstructed. Chest pain on exertion results from an increased demand for blood flow to the body and to the thickened heart muscle itself and may be worsened by distorted coronary arteries. Shortness of breath may be worse during exercise and also while lying down due to obstruction of blood flow and a leaky mitral valve, which cause a backflow of blood and a resulting increase in blood pressure and fluid around the lungs. In addition, individuals may experience palpitations due to abnormal heart rhythms resulting from abnormal electrical activity within the heart due to the thickened heart muscle and disorganized muscle cells.

\section{Complications}

As mentioned above, HOCM can lead to a number of life-threatening complications. In addition to abnormal heart rhythms and obstructed blood flow, individuals with HOCM may develop heart failure as the thickened heart muscle can become very stiff and unable to be filled with enough blood to then pump out and meet the body's needs. Backflow of blood through the mitral valve, known as mitral regurgitation, may also occur due to contact of the mitral valve with the thickened septum during each contraction, which can in turn worsen heart failure. Most seriously, abnormal heart rhythms, such as ventricular tachycardia and fibrillation, can result in sudden death.

\section{Investigations}

Echocardiography, which involves ultrasound imaging of the heart and may involve insertion of a flexible tube containing a transducer into the esophagus, is a commonly used imaging test as it can detect abnormally thick heart muscle as well as obstruction of blood flow and abnormal function of the heart's valves. In addition, an electrocardiogram (ECG), which involves attachment of electrodes to the skin to measure the heart's electrical activity, can detect abnormal heart rhythms as well as thickened heart muscle. Other imaging tests performed to evaluate the presence and severity of HOCM include cardiac MRI scans, Holter monitoring, which involves continuous ECG recordings over one to two days, and stress tests, which may use echocardiography to detect changes in blood flow or valve function caused by exercise. In addition, genetic testing should be carried out for those with family members known to have HOCM or who have a family history of sudden cardiac death.

\section{Management}

Treatment of HOCM centers on strategies to relieve symptoms, improve one's quality of life and importantly prevent sudden cardiac death in high-risk individuals. A number of medications, including beta-blockers and calcium channel blockers, may be used to decrease heart rate and the strength of heart contractions so that blood can be pumped to the body more efficiently. Interventions for HOCM include septal myectomy, which involves surgical removal of part of the thickened septum between the left and right ventricles, and septal ablation, which involves destruction of the thickened heart muscle by injection of alcohol through a thin tube into the artery that supplies that area. For those at high-risk of developing life-threatening abnormal heart rhythms, an implantable cardioverter-defibrillator (ICD) can be inserted into the chest, with electrodes being passed into a suitable vein and through to the heart under X-ray control. It continuously monitors one's heart rhythm and delivers electrical shocks in the event of a dangerous abnormal heart rhythm and can thus prevent sudden cardiac death. Risks of the procedure include bleeding, infection, formation of lung clots, and very small chances of a heart attack, stroke or death. As always, the risks should be weighed against the benefits and discussed with your surgeon or proceduralist. For more information, please visit the following websites: http://www.mayoclinic.org/diseases-conditions/hypertrophiccardiomyopathy/home/ovc-20122102

https://my.clevelandclinic.org/services/heart/disorders/hcm.aspx https://lifeinthefastlane.com/ecg-library/hcm/

http://www.4hcm.org/

http://www.utswmedicine.org/conditions-specialties/heart/ programs/general/hcm-hypertrophic-cardiomyopathy.html

doi: $10.21037 /$ acs.2017.07.06

Section Writer: Christopher Harris

Illustration Editor: Beth Croce

Section Editor: Stine Munkholm-Larsen
For specific information concerning your medical condition, ACS suggests that you consult your physician. This page may be photocopied non-commercially by physicians to share with patients. 